\title{
PROPOSTA DE MODELO DE REFERÊNCIA PARA UTILIZAÇÃO DA INTERNET DAS COISAS COMO SUPORTE À ESTRATÉGIA DE MARKETING.
}

\author{
Rafael Marques (rafael.marques @ polymtl.ca) - Polytechnique Montréal (Canadá) \\ Fabiano Armellini (fabiano.armellini@polymtl.ca) - Polytechnique Montréal (Canadá)
}

\section{RESUMO}

Agregar valor à seus negócios, é o que buscam empresas do mundo inteiro através do aumento da eficiência das atividades de marketing. A tendência abrange quase todos os setores, as decisões comerciais são tomadas a partir da percepção do mercado pelas empresas e de como devem usar essas informações para melhorar seus limites competitivos. Neste trabalho é definido o modelo genérico de estratégia de marketing que será aqui chamado de "marketing mix". Em seguida, são apresentados os diversos elementos IoT existentes e suas aplicações, seguido do o modelo de referência de IoT (Internet of Things) CISCO, com uma lista representativa de amplos fluxos de aparelhos, interações e tratamentos de dados que fornecem uma perspectiva sobre a divisão do campo de atuação da IoT. Em terceiro lugar é exposto um quadro de referência do potencial e localização da IoT para o marketing mix e como se dá o fluxo de transferência de informações do marketing mix no contexto da IoT. Com base na perspectiva da capacidade organizacional, este trabalho objetiva propor um modelo de referência que identifica e classifica as tecnologias de IoT, de forma a servir como ferramenta de suporte para uma estratégia de marketing, e ajudar na tomada de decisões gerenciais na adoção da correta tecnologia IoT. Finalmente, conclui-se com a sugestão de diversas problemáticas e contextos a serem abordados na pesquisa em curso.

Palavras chave: Internet of things; mix marketing; marketing em serviços; IoT; ferramenta de suporte ao marketing; marketing estratégico; Internet das coisas.

\section{Área: Gestão do Processo de Desenvolvimento de Produto}




\section{INVESTIGAÇÃO ATUAL DA IOT NO MARKETING}

A revolução está acontecendo através da Internet das Coisas (Internet of things - IoT) primeiramente terá um tremendo impacto no mundo como o conhecemos. A IoT, que envolve dispositivos, sistemas e serviços interconectados que dependem da comunicação autônoma de objetos físicos dentro da infraestrutura de Internet existente por exemplo (Atzori et al. 2010), trazem um conceito empolgante porque traz a inteligência da Internet para os produtos (Hoffman e Novak, 2015), tornando-os mais conectados e inteligentes (Nguyen e De Cremer, 2016). As aplicações do IoT abrangem várias áreas, tais como wearables, casas inteligentes, cidades inteligentes, automação industrial e muitos mais (por exemplo, Chuah et al., 2016). O IoT oferece grandes benefícios para inúmeras indústrias e sociedade como um todo $(\mathrm{Bi}, \mathrm{Xu}, \&$ Wang, 2014), com aplicações como implantes de monitoramento cardíaco, automóveis com sensores incorporados, transponders de biochip em animais de fazenda, dispositivos de busca e salvamento, ou sistemas de termostatos inteligentes e lavadoras / secadoras que utilizam Wi-Fi para monitoramento remoto (Kortuem et al., 2009; Porter e Heppelmann, 2014). Muitos dos sistemas e tecnologias IoT são novos e espera-se que o IoT inicie automação em quase todos os campos (por exemplo, Dholakia e Reyes, 2013). De acordo com Gartner (2015), haverá cerca de 20 bilhões de dispositivos no IoT até 2020.

Com essa rápida expansão e disseminação de seu impacto, é surpreendente que poucos estudos de marketing explorando o IoT tenham sido conduzidos, e que o assunto esteja sendo explorado quase de exclusivamente do ponto de vista tecnológico na literatura científica. Ainda existem muitas áreas de aplicação inexploradas, muitos desafios e questões que precisam ser melhoradas, e o impacto total para os grupos de partes interessadas está longe de ser claramente traçado. Por exemplo, para que o marketing possa efetivamente englobar a IoT, e como o IoT pode por si mesmo impulsionar as atividades de marketing.

O IoT tem sido proclamado como essencial para a inovação organizacional, adaptação e sucesso, especialmente para empresas com altas quantidades de conectividade, rede e dados (Jones et al. 2013; Yu et al. 2016). No entanto, são necessárias mais pesquisas para explorar as capacidades necessárias para adotar o IoT na organização e como elas se relacionam com diferentes aspectos do marketing, particularmente para a organização orientada a relacionamento com o cliente, alianças, joint ventures e parcerias. Muitas das regras de marketing estão mudando e muitas novas abordagens serão introduzidas nesta nova era IoT.

\section{CRITÉRIOS DE SELEÇÃO DO MODELO DE MARKETING}

O Marketing apoiado pela IoT é um campo recém-nascido. Organizações como a Apple apenas começaram campanhas de marketing para seus produtos. No entanto, podem ser estabelecidos paralelos entre a comercialização de produtos e serviços de IoT e a de produtos de nova tecnologia que vieram antes, como computadores pessoais e telefones inteligentes. A premissa para desenhar tal paralelo está nas semelhanças inerentes dos produtos e dos consumidores a que servem. IoT são produtos de alta tecnologia, o que também é o caso de novos produtos de tecnologia. Em segundo lugar, o IoT envolve um elemento de inovação disruptiva (Ma e Zhang, 2011), da mesma forma, os telefones inteligentes impactaram nas comunicações (Conole, de Laat et al. 2008), os produtos IoT estão posicionados para impactar muitas indústrias. Um estudo de Bohli, Sorge e Westhoff (2010) mostrou que os consumidores de produtos IoT apresentam comportamento semelhante ao de produtos como PCs e telefones inteligentes em termos de disposição para pagar, lealdade e aceitação da tecnologia. 
O'Connor e Veryzer (2001) confirmaram que ligar as oportunidades de mercado às novas tecnologias era um elemento crítico na comercialização bem-sucedida. Eles também descobriram que, uma vez identificadas, essas oportunidades precisam ser apoiadas por gerentes e tecnólogos em organizações, a fim de que a tecnologia apresente um design comercialmente viável. $\mathrm{O}$ presente artigo pesquisa explora os parâmetros relevantes que são importantes para todas as partes interessadas e ajuda a auxiliar os negócios baseados em IoT a ganhar 'usuários (clientes) no segmento.

Para alinhar as estratégias de marketing às ferramentas de IoT, faz-se necessário delimitar as estratégias de marketing condensando as inúmeras estratégias existentes na literatura, pois a evolução do marketing estratégico como campo de estudo, ao longo das últimas décadas, pode ser vista como uma confluência de perspectivas, paradigmas, teorias, conceitos, estruturas, princípios, métodos, modelos e métricas a partir de uma série de áreas de estudo, empresas e consumidores.

A Associação Americana de Marketing (AMA) Grupo de Interesse Especial em Estratégia de Marketing (SIG), em seu convite para nomeações para o Mahajan Award for Lifetime Contributions to Marketing Strategy Research, definiu o domínio de pesquisa de estratégia de marketing de forma ampla, para incluir todas as questões de marketing estratégico da empresa, decisões e problemas (AMA, 2009).

No entanto, existem algumas questões relacionadas com a delimitação do domínio da pesquisa de estratégia de marketing. Primeiramente, incluir os problemas de todos os níveis da empresa no marketing estratégico ou incluir todos os problemas do marketing, decisões e problemas em cada nível da empresa, são coisas muito diferentes. Em segundo lugar, definir o domínio da pesquisa de estratégia de marketing como incluindo apenas questões de marketing estratégico da empresa, decisões e problemas está claramente em desacordo com o corpo cumulativo de literatura sobre marketing estratégico.

Dadas as circunstâncias, qualquer tentativa de aumentar o campo intelectual da pesquisa para abranger uma maior parcela das escolas do marketing estratégico é inerentemente fútil. Quaisquer que sejam os limites estabelecidos, inevitavelmente omitirá pesquisadores cujo trabalho deve ser incluído. No entanto, quando delimitamos os limites adotando um modelo de ampla aceitação, inevitavelmente são incorporados alguns que de outra forma teríamos excluído.

"Definir os limites de um campo de investigação pode, a longo prazo, ser apenas um gesto, mas, para começar, a delimitação, por mais provisória que seja, é indispensável. O perigo não é muito grande se tivermos em mente, que os limites que estabelecemos são um auxílio à compreensão." (Inkeles 1964, p.1)

As observações de Inkeles acima servem para destacar a necessidade e a importância de delinear o domínio de qualquer campo de estudo, os desafios e limitações de cada empreendimento, e a necessidade de revisar periodicamente a questão, dada a natureza evolutiva dos campos de estudo.

Neste contexto, o restante deste trabalho está organizado da seguinte forma: Em primeiro lugar, é definido o modelo genérico de estratégia de "marketing mix". Em seguida, é apresentado o modelo de referência de IoT, uma lista representativa de amplos fluxos de aparelhos, interações e tratamentos de dados que fornecem uma perspectiva sobre a divisão do campo de atuação da IoT. Em terceiro lugar é sugerido um quadro de referência do potencial da IoT para o marketing. Finalmente, o estudo conclui-se com a elaboração de um roteiro para análise e aplicações específicas da IoT nesse contexto. 


\section{O CONCEITO DO MARKETING MIX}

Borden afirma ser o primeiro a usar o termo "marketing mix" e que foi sugerido pela descrição de Culliton (1948) um executivo de negócios como "misturador de ingredientes". No entanto, Borden não definiu formalmente o mix de marketing, para ele, simplesmente consiste de elementos ou ingredientes importantes que compõem um programa de marketing (Borden, 1965, p.368). McCarthy (1964, p.35) refinou isso mais ainda e definiu o mix de marketing como uma combinação de todos os fatores no comando de um gerente de marketing para satisfazer o mercado-alvo. Mais recentemente, McCarthy e Perreault (1987) definiram o mix de marketing como as variáveis controláveis que uma organização pode coordenar para satisfazer seu mercado-alvo. Esta definição (com pequenas mudanças) é amplamente aceita como pode ser visto pela definição do mix de marketing por Kotler e Armstrong: como o conjunto de variáveis de marketing controláveis que a empresa mistura para produzir a resposta que deseja no mercado-alvo (1989, p.45).

A essência do conceito de mix de marketing é, portanto, a idéia de um conjunto de variáveis controláveis ou um "kit de ferramentas" (Shapiro, 1985) à disposição da gerência de marketing que pode ser usada para influenciar os clientes. O desacordo na literatura é sobre o que essas variáveis ou ferramentas são.

Kopler e Armstrong (2013), por sua vez adicionaram o quinto "P", quando incluiram pessoas ao marketing mix em seu livro Principles of marketing (ver Kotler e Armstrong, 1989)

Foram sugeridas várias modificações, porém $\mathrm{O}$ mais influente dos modelos teóricos alternativos, são os 7Ps de (Booms e Bitner, 1981) mix onde sugerem que não só os tradicionais 4 ou 5 "P's" precisam ser modificados para serviços, mas também precisam ser estendidos para incluir participantes, evidência física e processo. Sua estrutura é discutida na Tabela 1.

Embora constatarmos que havia uma grande insatisfação com o quadro de 4Ps, foi mais difícil avaliar o quão bem o quadro de Booms e Bitner foi aceito como um quadro geral para o marketing. No entanto, uma pesquisa realizada por (Rafiq and Ahmed 1995) no Reino Unido e na Europa nos permitiu avaliar não só a aceitação da estrutura de 7Ps, mas também quão bem as novas variáveis de participantes, processo e evidência física foram aceitos. A pesquisa mostra mostra que todas as variáveis de 7Ps foram consideradas como tendo pelo menos alguma relevância para todos os tipos de marketing, incluindo marketing introdutório e de consumo, onde se poderia esperar maior aderência ao quadro de 4Ps. No entanto, como seria de esperar, há uma diferença na ênfase no uso das variáveis de mix para diferentes tipos de marketing. Por exemplo, há uma ênfase forte nos 4Ps tradicionais no marketing do consumidor e menos ênfase em outras variáveis do mix. Da mesma forma, os participantes e processos recebem mais ênfase no marketing de serviços do que em outros tipos de marketing.

Os resultados apresentados na pesquisa de (Rafiq e Ahmed 1995) sugerem que a estrutura 4Ps é pensada para ser mais relevante para marketing introdutório e marketing de consumo. $\mathrm{O}$ resultado também sugere que o quadro de 7Ps já alcançou um alto grau de aceitação como um mix de marketing genérico entre os acadêmicos de renome estudados. 
Tabela 1 4p's versus 7p's (Rafiq e Ahmed 1995)

\begin{tabular}{|c|c|c|c|c|c|c|}
\hline Product & Pomoção & Price & Praça & Pessoas & Processos & Evidências físicas \\
\hline \multicolumn{7}{|c|}{$4 P^{\prime}$ s Tradicionais } \\
\hline Variedade & Canais de distribui ção & Nível do preço & Propaganda & & & \\
\hline Qualidade & Cobertura & Descontos & Vendas & & & \\
\hline Design & Inventário & $\begin{array}{c}\text { Condições de } \\
\text { pagamento }\end{array}$ & $\begin{array}{l}\text { Prospecção de } \\
\text { mercado }\end{array}$ & & & \\
\hline Marca & Logistica & & Rel açỗes públicas & & & \\
\hline \multicolumn{7}{|l|}{ Embalagem } \\
\hline \multicolumn{7}{|l|}{ Source: Kotler (1976) } \\
\hline \multicolumn{7}{|c|}{ Mix Marketing modificado e expandido para os $7 p$ 's } \\
\hline Product & Promoção & Peço & Raça & Pessoas & Processos & Evidências físicas \\
\hline Qualidade & $\begin{array}{l}\text { Comuni cação de } \\
\text { marketing }\end{array}$ & Posicionamento & Canais de distribuição & $\begin{array}{l}\text { Pessoas implicadas } \\
\text { nas atividades do } \\
\text { marketing }\end{array}$ & Foco no diente & $\begin{array}{l}\text { Conhecimento } \\
\text { adqui rido do negócio }\end{array}$ \\
\hline Marca & Institucional & Acessibilidade & CMR & $\begin{array}{l}\text { Atendimento } \\
\text { personalisado }\end{array}$ & $\begin{array}{c}\text { Prospecção de } \\
\text { negócios }\end{array}$ & Embalagem \\
\hline Imagem & Vendas & Canais de distribuição & Vendas e prom oção & Recrutamento & Suporte de $\mathrm{Ti}$ & Experiencia On-line \\
\hline Mix de produtos & Branding & $\begin{array}{l}\text { Capacidade de } \\
\text { distribuiçã̃o }\end{array}$ & $\begin{array}{c}\text { Segmentação dos } \\
\text { canais }\end{array}$ & Incentivos & Design & $\begin{array}{l}\text { Envolvimento do } \\
\text { cliente }\end{array}$ \\
\hline Capacidades & Marketing direto & Descontos & & Aparencia & Inovação & Fluxo de atividades \\
\hline Suporte & Interação & Valor agregado & & $\begin{array}{l}\text { Relações Inter- } \\
\text { pessoais }\end{array}$ & $\begin{array}{c}\text { Pesquisae } \\
\text { Desenvolyimento }\end{array}$ & \\
\hline Serviço à clientela & & $\begin{array}{l}\text { Condições de } \\
\text { pagamento }\end{array}$ & & Postura & & \\
\hline Disponibilidade & & & & Cultura & & \\
\hline Diferenciação & & & & & & \\
\hline
\end{tabular}

A Figura 1 ilustra o fato de que na comercialização de bens, os participantes, infra-estrutura e partes do processo do mix de marketing estendido são normalmente delegados aos distribuidores. Isso ocorre porque normalmente esses elementos fazem muito pouca diferença para a qualidade do produto entregue no mercado de bens de consumo e o que é importante é a ampla distribuição. Quando a qualidade ou a imagem do produto for afetada, os intermediários podem ser eliminados. Isto é particularmente provável ocorrer se o fabricante produz uma gama profunda de produtos. (Rafiq e Ahmed 1995). Um exemplo claro deste processo é quando o fabricante tem suas próprias lojas de varejo para enfatizar a exclusividade de seus produtos e garantir que os produtos tenham a qualidade que os clientes esperam da marca. A eliminação de intermediários, ou pelo menos o encurtamento do canal de distribuição, também é provável que ocorra quando o produto requer um alto grau de serviço.

Em contraste, na comercialização de serviços, todas as partes do mix estão normalmente sob o controle direto dos prestadores de serviços (ver Figura 1). Isto é, os serviços são menos frequentemente distribuídos através de intermediários. No entanto, alguns serviços são distribuídos através de intermediários (por exemplo, organização de feiras, seguros, etc.). Isto tem maior probabilidade de ocorrer quando um serviço padronizado pré-embalado pode ser oferecido. McDonald's, Petro-Canadá, Pizza Dominos fornecem bons exemplos de onde um produto padronizado é distribuído através de um intermediário (ou seja, um franqueado). Em todos estes exemplos, os produtores mantêm um controlo rigoroso sobre o produto, a promoção, os preços cobrados, a localização física, o aspecto, os procedimentos e padrões operacionais. Isto só é possível porque os produtores são capazes de estabelecer especificações exatas para 
os seus produtos. Assim, onde a prestação de serviços pode ser padronizada e a qualidade do serviço facilmente controlada e monitorada, o marketing de serviços se assemelha ao marketing de mercadorias. De fato, os profissionais de marketing de serviço devem procurar ativamente padronizar seus serviços ou "industrializar" os serviços como coloca Levitt (1976). Mesmo nestes exemplos, os canais de distribuição tendem a ser curtos. Uma análise semelhante pode ser estendida a outros tipos de marketing.

Fig. 01 - O marketing mix expandido e a relação entre produtores e intermediários na comercialização de bens e serviços (Fonte: Rafiq e Ahmed 1995)
Fornecedor de bens materiais
Intermediários
Forncedor de serviços

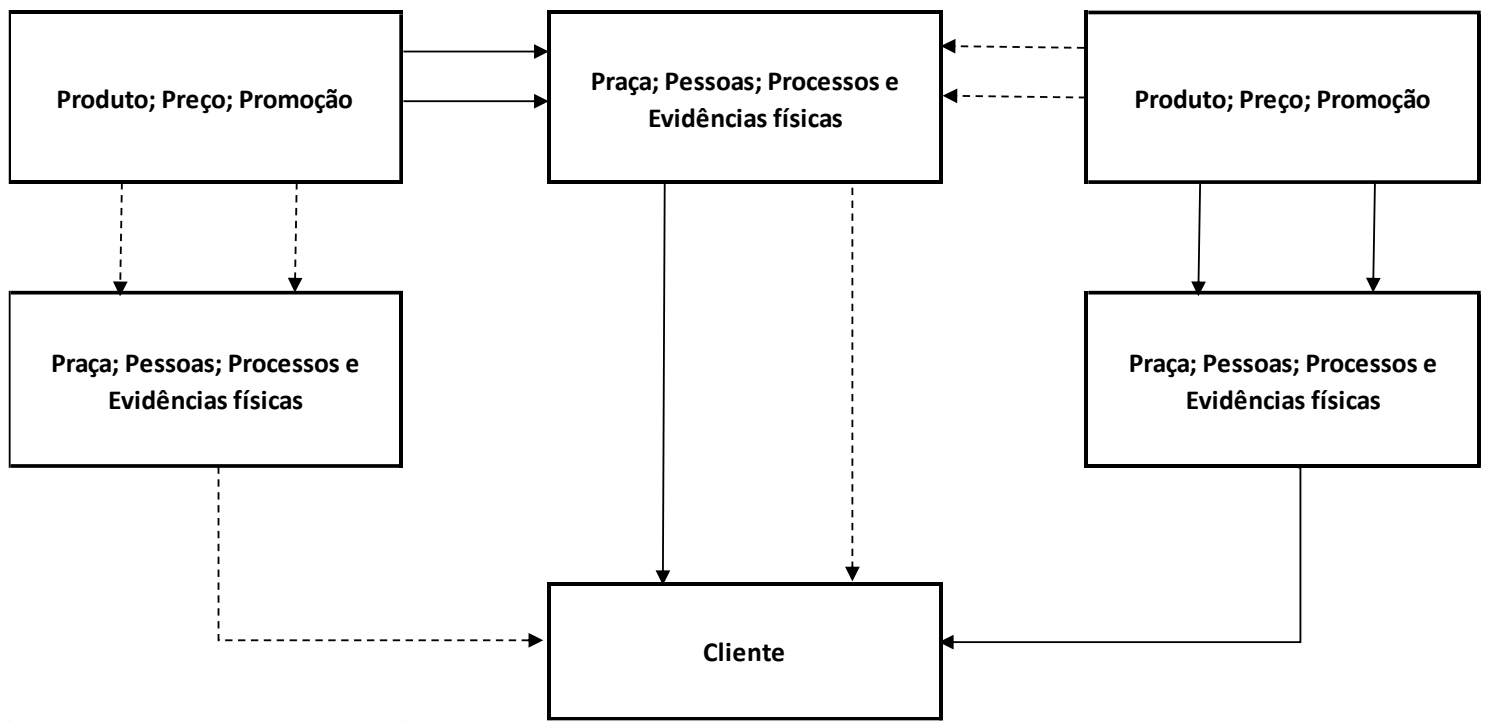

Rota frequente

Rota menos frequente

\section{UM MODELO DE REFERÊNCIA DE IOT}

Atualmente, muitos pesquisadores estudam a IoT. O conceito básico do IoT é que várias coisas ou objetos estão interligados uns com os outros e alcançam seu objetivo comum exemplo (Atzori et al. 2010).

Vários tipos de coisas ou objetos podem ser interligados através da utilização de tecnologias de comunicação sem fios, tais como a identificação por radiofrequência (RFID) ou near field communication (NFC) (Atzori et al. 2010 e Gluhak et al. 2011). Por exemplo, no domínio da logística, os investigadores examinaram a redução da logística através da compra de materiais, transporte e armazenamento utilizando o IoT (Bendavid e Boeck 2011). As pessoas também consideraram métodos eficientes que analisam informações pessoais, como atividades sociais ou históricas na web usando dispositivos inteligentes no IoT (Welbourne et al. 2009)

Conforme identificado por Atzori et al. (2010), a IoT pode ser realizada em três paradigmas: orientado para a internet (middleware), orientado a coisas (sensores) e orientado à semântica (conhecimento). Embora este tipo de delineamento seja exigido devido à natureza interdisciplinar do assunto, a utilidade do IoT pode ser desencadeada somente em um domínio de aplicação onde os três paradigmas se cruzam. O grupo RFID define a Internet de Coisas 
como "A rede mundial de objetos interligados de endereço exclusivo, com base em protocolos de comunicação padrão". De acordo com o Cluster de projetos de pesquisa europeus na Internet de Coisas (H. Sundmaeker et al. 2010), "Coisas" são participantes ativos em negócios, informações e processos sociais onde eles são capazes de interagir e se comunicar entre si e com o meio ambiente, trocando dados e informações detectadas sobre o ambiente, ao mesmo tempo que reage de forma autônoma aos eventos reais/físicos e influenciando-o executando processos que desencadeiam ações e criam serviços com ou sem intervenção humana direta.

De acordo com a Forrester research (Forester Research, 2010), um ambiente inteligente usa tecnologias de informação e comunicação para tornar mais conscientes, interativos e eficientes os componentes e serviços de infra-estrutura crítica da administração, educação, saúde, segurança pública, imóveis, transporte e serviços públicos de uma cidade.

Nossa visão da Internet de Coisas para ambientes inteligentes é a interligação de dispositivos de detecção e atuação que oferecem a capacidade de compartilhar informações de marketing estratégico através de uma estrutura unificada, desenvolvendo uma imagem operacional comum permitindo o desenvolvimento de estratégias inovadoras. Isto é conseguido por detecção ubíqua perfeita, análise de dados e representação de informação com o Cloud computing como a estrutura unificadora.

\subsection{Elementos IoT}

Aqui é apresentado uma taxonomia que ajudará na definição dos componentes necessários para a Internet das Coisas de uma perspectiva de alto nível. Taxonomias específicas de cada componente podem ser encontradas em outro lugar (S. Tilak et al. 2002, Tory e Moller $2004 \mathrm{e}$ R. Buyya et al. 2011). Existem três componentes IoT que permitem a localização perfeita: (a) Hardware composto de sensores, atuadores e hardware de comunicação incorporado (b) Ferramentas de armazenamento e computação middleware on demand para análise de dados e (c) Apresentação e novela de fácil compreensão de visualização e ferramentas de interpretação que podem ser amplamente acessadas em diferentes plataformas e que podem ser projetadas para diferentes aplicativos.

Nesta seção, discutimos algumas tecnologias habilitadoras nessas categorias que compõem os três componentes mencionados acima.

\subsubsection{Radio Frequency Identification (RFID)}

A tecnologia RFID é um grande avanço no paradigma de comunicação incorporado que permite o design de microchips para comunicação de dados sem fio. Eles ajudam na identificação automática de qualquer coisa que eles estão ligados para atuar como um código de barras eletrônico (Welbourne et al. 2009, Juels 2006). As etiquetas RFID passivas não são alimentadas por bateria e usam a energia do sinal de interrogação do leitor para comunicar a ID ao leitor de RFID. Isso resultou em muitas aplicações, particularmente no varejo e na gestão da cadeia de suprimentos. As aplicações podem ser encontradas no transporte (substituição de bilhetes, adesivos de registro) e aplicações de controle de acesso também. As tags passivas estão sendo usadas atualmente em muitos cartões bancários e etiquetas de pedágio que estão entre as primeiras implantações globais. Os leitores RFID ativos possuem seu próprio suprimento de bateria e podem instanciar a comunicação. Das várias aplicações, a principal aplicação de etiquetas RFID ativas está em recipientes portuários (Juels, 2006) para monitorar a carga. 


\subsubsection{Wireless Sensor Networks (WSN)}

Os recentes avanços tecnológicos em circuitos integrados de baixa potência e comunicação sem fio disponibilizaram dispositivos em miniatura eficientes, de baixo custo e de baixa potência para uso em aplicações de sensoriamento remoto. A combinação desses fatores melhorou a viabilidade de utilizar uma rede de sensores consistindo de um grande número de sensores inteligentes, permitindo a coleta, processamento, análise e disseminação de informações valiosas, reunidas em diversos ambientes (Akyildiz et al., 2002). O RFID ativo é quase o mesmo que os nós WSN de extremidade inferior com capacidade de processamento e armazenamento limitados. Os desafios científicos que devem ser superados para realizar o enorme potencial das WSNs são substanciais e multidisciplinares na natureza (Akyildiz et al., 2002). Os dados do sensor são compartilhados entre nós de sensores e enviados para um sistema distribuído ou centralizado para análise.

\subsubsection{Esquemas de endereçamento}

A capacidade de identificar exclusivamente as "Coisas" é fundamental para o sucesso do IoT. Isso não só nos permitirá identificar exclusivamente bilhões de dispositivos, mas também controlar dispositivos remotos através da Internet. Os recursos mais críticos da criação de um endereço exclusivo são: singularidade, confiabilidade, persistência e escalabilidade. Todos os elementos que já estão conectados e aqueles que serão conectados devem ser identificados por sua identificação, localização e funcionalidades únicas.

\subsubsection{Armazenamento e análise de dados}

Um dos resultados mais importantes deste campo emergente é a criação de uma quantidade de dados sem precedentes. $\mathrm{O}$ armazenamento, a propriedade e a caducidade dos dados tornam-se questões críticas. A Internet consome até $5 \%$ da energia total gerada hoje e com esses tipos de demandas, certamente aumentará ainda mais. Assim, os centros de dados que funcionam com energia colhida e estão centralizados garantirão a eficiência energética e a confiabilidade. Os dados devem ser armazenados e utilizados de forma inteligente para monitoramento e atuação inteligentes. É importante desenvolver algoritmos de inteligência artificial que possam ser centralizados ou distribuídos com base na necessidade. Novos algoritmos de fusão precisam ser desenvolvidos para dar sentido aos dados coletados. Métodos de aprendizagem de máquinas temporais não-lineares de última geração baseados em algoritmos evolutivos, algoritmos genéticos, redes neurais e outras técnicas de inteligência artificial são necessários para a tomada automática de decisões. Esses sistemas apresentam características como interoperabilidade, integração e comunicação adaptativa. Eles também possuem uma arquitetura modular, tanto em termos de design do sistema de hardware quanto de desenvolvimento de software, e geralmente são muito adequados para aplicações IoT. Mais importante ainda, é necessária uma infraestrutura centralizada para suporte de armazenamento e análise. Isso forma a camada de middleware do IoT e há inúmeros desafios envolvidos que são discutidos em seções futuras. A partir de 2012, as soluções de armazenamento baseadas em nuvem estão se tornando cada vez mais populares e nos próximos anos, análises e visualizações baseadas em nuvem estão previstas plataformas.

\subsubsection{Visualização}

A visualização é fundamental para uma aplicação IoT, pois isso permite a interação do usuário com o meio ambiente. Com os recentes avanços nas tecnologias de tela sensível ao toque, o uso de tablets e telefones inteligentes tornou-se muito intuitivo. Para que um leigo se beneficie plenamente da revolução IOT, é necessário criar uma visualização atrativa e fácil de entender. 
À medida que passamos das telas 2D para 3D, mais informações podem ser fornecidas de forma significativa para os consumidores. Isso também permitirá que os decisores políticos convertam dados em conhecimento, o que é crítico na rápida tomada de decisões. A extração de informações significativas a partir de dados brutos não é trivial. Isso engloba a detecção de eventos e a visualização dos dados crus e modelados associados, com informações representadas de acordo com as necessidades do usuário final.

\section{O MODELO DE REFERÊNCIA DA CISCO}

Como visto, pode-se utilizar o IoT em vários domínios e o IoT tem grande potencial como um novo paradigma de telecomunicações sem fio modernas. Neste trabalho, em particular, é considerado o IoT em um modelo de referência elaborado pela Cisco $^{\mathrm{TM}}$. Este modelo foi desenvolvido com a finalidade de fornecer definições e descrições claras que possam ser aplicadas com precisão aos elementos e funções dos sistemas e aplicações IoT. O modelo ajuda a quebrar os sistemas complexos expostos aqui anteriormente para que cada parte seja mais compreensível, e também fornece informações adicionais para identificar com precisão os níveis da IoT e estabelecer uma relação com o marketing mix. Através deste modelo é possível também identificar onde os tipos específicos de processamento são otimizados em diferentes partes do sistema. Com isso é fornecido um primeiro passo para permitir que os profissionais de marketing criem produtos IoT que funcionam entre si, tornando a IoT real e acessível, em vez de simplesmente conceitual.

Em um sistema IoT, os dados são gerados por vários tipos de dispositivos, processados de diferentes maneiras, transmitidos para diferentes locais e movidos por aplicativos. O modelo de referência IoT proposto é composto de sete níveis. Cada nível é definido com terminologia que pode ser padronizada para criar um quadro de referência. O Modelo de Referência IoT não restringe o escopo ou a localidade de seus componentes. Por exemplo, a partir de uma perspectiva física, cada elemento poderia residir em um único rack de equipamentos ou poderia ser distribuído em todo o mundo.

As redes de comunicação de dados tradicionais têm múltiplas funções, como evidenciado pelo modelo de referência de 7 camadas da Organização Internacional de Normalização (ISO). No entanto, um sistema IoT completo contém muitos níveis, além da rede de comunicações. O Modelo de Referência IoT também permite que o processamento que ocorre em cada nível varie de trivial para complexo, dependendo da situação. O modelo descreve como as tarefas em cada nível devem ser tratadas para manter a simplicidade, permitir alta escalabilidade e garantir suporte. Finalmente, o modelo define as funções necessárias para que um sistema IoT seja completo. (CISCO, 2014)

A Figura 2 ilustra o modelo de referência IoT e seus níveis. É importante notar que, no IoT, os dados fluem em ambas as direções. Em um padrão de controle, as informações de controle fluem da parte superior do modelo (nível 7) para a parte inferior (nível 1).

Em um padrão de monitoramento, o fluxo de informações é o inverso. Na maioria dos sistemas, o fluxo será bidirecional. 

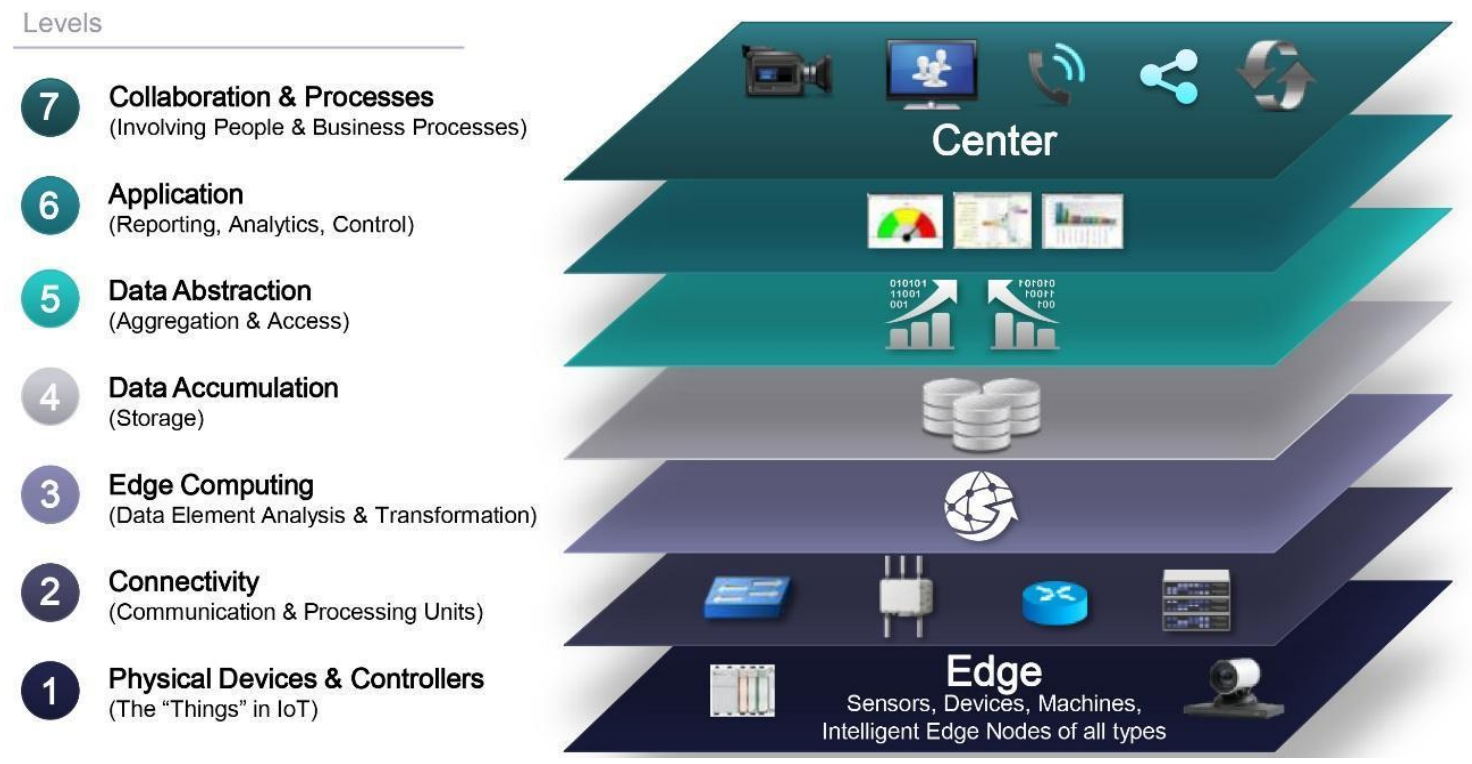

\subsection{Nível 1: Dispositivos físicos e controladores}

O Modelo de Referência IoT começa com o Nível 1: dispositivos físicos e controladores que podem controlar vários dispositivos. Estas são as "coisas" da internet das coisas, e eles incluem uma ampla gama de dispositivos de terminal que enviam e recebem informações. Hoje, a lista de dispositivos já é extensa. Tornar-se-á quase ilimitado enquanto mais equipamento é adicionado ao IoT ao longo do tempo.

\subsection{Nível 2: Conectividade}

As comunicações e conectividade estão concentradas no nível 2. A função mais importante do Nível 2 é a transmissão de informações confiável e oportuna. Isso inclui transmissões entre dispositivos (Nível 1) e a rede, entre a rede (Nível 2) e o processamento de informação de baixo nível ocorrendo no Nível 3 e através das redes (leste-oeste).

\subsection{Nível 3: Computação em Borda (Nevoeiro)}

As funções do Nível 3 são impulsionadas pela necessidade de converter fluxos de dados de rede em informações que sejam adequadas para armazenamento e processamento de nível superior no Nível 4 (acumulação de dados). Isso significa que as atividades de Nível 3 se concentram em análise e transformação de dados de alto volume. Por exemplo, um dispositivo de sensor Nível 1 pode gerar amostras de dados várias vezes por segundo, 24 horas por dia, 365 dias por ano. Um princípio básico do Modelo de Referência do IoT é que o sistema mais inteligente inicia o processamento da informação tão cedo quanto possível da fronteira da rede. Isso às vezes é referido como computação em névoa. No nível 3 é onde isso ocorre. Visto que os dados são normalmente submetidos ao equipamento de rede de nível de conectividade (Nível 2) por dispositivos em unidades pequenas, o processamento de Nível 3 é realizado numa base de pacote por pacote. Esse processamento é limitado, porque há apenas consciência de unidades de dados e não "sessões" ou "transações". O processamento de Nível 3 pode abranger muitos exemplos, tais como:

- Avaliação: Avaliação de dados para determinar se deve ser processado a um nível superior; 
- Formatação: reformulação de dados para processamento consistente de nível superior;

- Expansão / decodificação: manipulação de dados crípticos com contexto adicional (como a origem);

- Destilação / redução: Reduzir e / ou resumir dados para minimizar o impacto dos dados e do tráfego na rede e sistemas de processamento de nível superior;

- Avaliação: determinar se os dados representam um limiar ou alerta; Isso pode incluir o redirecionamento de dados para destinos adicionais

\subsection{Nível 4: Acumulação de dados}

Os sistemas de rede são construídos para mover dados com segurança. Os dados estão "em movimento". Antes do Nível 4, os dados estão se movendo pela rede à taxa e organização determinada pelos dispositivos que geram os dados. O modelo é orientado a eventos. Conforme definido anteriormente, os dispositivos de Nível 1 não incluem recursos de computação. No entanto, algumas atividades computacionais podem ocorrer no nível 2 , como tradução de protocolo ou aplicação da política de segurança de rede. Outras tarefas de computação podem ser executadas no nível 3, como a inspeção de pacotes. Conduzir tarefas computacionais o mais próximo possível da borda do IoT, com sistemas heterogêneos distribuídos em vários domínios de gerenciamento, representa um exemplo de computação em nevoeiro. Os serviços de computação em névoa e nevoeiro são uma característica distintiva do IoT.

A maioria dos aplicativos não pode, ou não precisa, processar dados na velocidade da rede. As aplicações normalmente assumem que os dados estão "em repouso" - ou imutáveis - na memória ou no disco. No Nível 4, Acumulação de Dados, os dados em movimento são convertidos em dados em repouso. O nível 4 determina:

- Se os dados são de interesse para níveis mais elevados: Se assim for, o processamento de Nível 4 é o primeiro nível configurado para atender as necessidades específicas de um nível superior.

- Se os dados devem ser persistentes: Os dados devem ser mantidos no disco em um estado não-volátil ou acumulados na memória para uso de curto prazo?

- O tipo de armazenamento necessário: A persistência requer um sistema de arquivos, um grande sistema de dados ou um banco de dados relacional?

- Se os dados estão organizados corretamente: Os dados estão organizados de forma apropriada para o sistema de armazenamento necessário?

- Se os dados devem ser recombinados ou recalculados: os dados podem ser combinados, recalculados ou agregados com informações previamente armazenadas, algumas das quais podem ter sido provenientes de fontes não-IoT.

Como o Nível 4 captura dados e o coloca em repouso, torna-se possível a utilização desses dados por aplicativos em uma base de tempo não-real. Os aplicativos acessam os dados quando necessário. Em suma, o Nível 4 converte dados baseados em eventos para processamento baseado em consulta. Este é um passo crucial para superar as diferenças entre o mundo de redes em tempo real e o mundo de aplicações não em tempo real.

\subsection{Nível 5: Abstração de Dados}

Os sistemas IoT precisarão escalar para um nível corporativo ou mesmo global e exigirão vários sistemas de armazenamento para acomodar os dados e dados de dispositivos IoT dos sistemas 
ERP, HRMS, CRM e outros sistemas empresariais tradicionais. As funções de abstração de dados do Nível 5 são focadas em renderizar dados e seu armazenamento de maneira que possibilite o desenvolvimento de aplicativos mais simples e melhorados.

Com vários dispositivos gerando dados, existem muitas razões pelas quais esses dados não podem aterrar no mesmo armazenamento de dados:

- Pode haver demasiados dados para colocar em um só lugar.

- Transferir dados para um banco de dados pode consumir muito poder de processamento, de modo que recuperá-lo deve ser separado do processo de geração de dados. Isso é feito hoje com o processamento de transações on-line (OLTP)

- Bancos de dados e data warehouses.

- Os dispositivos podem estar separados geograficamente e o processamento é otimizado localmente.

- Os níveis 3 e 4 podem separar "fluxos contínuos de dados brutos" de "dados que representam um evento".

- O armazenamento de dados para dados em fluxo contínuo pode ser um grande sistema de dados, como o Hadoop. O armazenamento para dados de eventos pode ser um sistema de gerenciamento de banco de dados relacional (RDBMS) com tempos de consulta mais rápidos.

- Diferentes tipos de processamento de dados podem ser necessários. Por exemplo, o processamento na loja se concentrará em coisas diferentes do processamento de resumo em todas as lojas.

Por estas razões, o nível de abstração de dados deve processar muitas coisas diferentes. Esses incluem:

- Conciliar vários formatos de dados de diferentes fontes;

- Assegurar uma semântica consistente de dados entre fontes;

- Confirmar que os dados estão completos para o aplicativo de nível superior.

\subsection{Nível 6: Aplicação}

Nível 6 é onde a interpretação de informações ocorre. O software nesse nível interage com o Nível 5 e os dados em repouso, para que ele não precise operar em velocidades de rede. $\mathrm{O}$ Modelo de Referência IoT não define estritamente uma aplicação. As aplicações variam com base nos mercados verticais, na natureza dos dados do dispositivo e nas necessidades do negócio. Por exemplo, alguns aplicativos se concentrarão no monitoramento de dados do dispositivo. Alguns se concentrarão no controle de dispositivos. Alguns combinarão dados de dispositivo e não de dispositivo. As aplicações de monitoramento e controle representam muitos modelos de aplicativos, padrões de programação e pilhas de software diferentes, levando a discussões de sistemas operacionais, mobilidade, servidores de aplicativos, hypervisors, multithreading, multi-tenancy, etc. Esses tópicos estão além do escopo de nossa discussão do Modelo de Referência da IoT. Basta dizer que a complexidade da aplicação variará amplamente.

Exemplos incluem:

- Aplicações empresariais de missão crítica, como ERP generalizado ou soluções de indústria especializada; 
- Aplicativos móveis que lidam com interações simples;

- Relatórios de Business Intelligence, onde o aplicativo é o servidor de BI;

- Aplicações analíticas que interpretam dados para decisões empresariais;

- As aplicações de gerenciamento de sistemas / centros de controle que controlam o próprio sistema IoT e não atuam sobre os dados produzidos por ele.

Se os Níveis 1-5 forem adequadamente arquitetados, a quantidade de trabalho exigida pelo Nível 6 será reduzida. Se o Nível 6 for projetado adequadamente, os usuários serão capazes de fazer seu trabalho melhor.

\subsection{Nível 7: Colaboração e Processos}

Uma das principais distinções entre a Internet das Coisas (IoT) e o sistema IoT é que o IoT inclui pessoas e processos. Esta diferença torna-se particularmente clara no Nível 7: Colaboração e Processos. O sistema IoT, e as informações que ele cria, é de pouco valor a menos que produza ação, o que muitas vezes requer pessoas e processos.

Os aplicativos executam lógica de negócios para capacitar as pessoas. As pessoas usam aplicativos e dados associados para suas necessidades específicas. Muitas vezes, várias pessoas usam o mesmo aplicativo para uma variedade de propósitos diferentes. Portanto, o objetivo não é a aplicação e sim capacitar as pessoas a fazer melhor seu trabalho. Os aplicativos (nível 6) dão aos empresários os dados certos, no momento certo, para que eles possam fazer a coisa certa.

Mas freqüentemente, a ação necessária requer mais de uma pessoa. As pessoas devem ser capazes de se comunicar e colaborar, às vezes usando a Internet tradicional, para tornar o IoT útil. Comunicação e colaboração muitas vezes requer múltiplos passos. E geralmente transcende múltiplas aplicações. É por isso que o Nível 7, representa um nível mais alto no lugar de uma única aplicação.

\section{QUADRO DE REFERÊNCIA DO POTENCIAL DA IOT PARA O MARKETING}

A decisão estratégica nas empresas é o mecanismo que é usado para alinhar a estratégia de uma empresa com seu objetivo competitivo e ambiente (Ward e Duray, 2000) e é determinado pelas condições de restrição externa e pelas preferências dos tomadores de decisão (Rui H e Yip GS, 2008). De acordo com a perspectiva estratégica, consideramos o marketing estratégico da IoT como a resposta positiva das empresas ao ambiente IoT, a fim de obter vantagem competitiva usando tecnologias avançadas de IoT. No contexto do uso de IoT nos negócios, as restrições externas são principalmente das forças da tecnologia relacionada com IoT push e market pull (Haller et al. 2009 e Welbourne E et al 2009). Enquanto isso, a preferência do tomador de decisão indica a intenção estratégica do gestor para o posicionamento de marketing no negócio da IoT.

A Figura 3 mostra a estrutura do modelo de marketing mix para aplicações IoT derivadas da literatura (seções 4 e 5) e consultas com profissionais do domínio de marketing e tecnologia.

A figura mostra os blocos de construção que constituem um modelo de marketing da IoT com os 7P's (Produto, Preço, Promoção, Praça, Pessoas, Processos e Evidências físicas) e os setores para cada bloco de construção. Neste modelo, foram analisados 3 setores. O primeiro setor é orientado para os produtores. O segundo setor é orientado para o transporte, distribuidores, logística e desenvolvimento de software e interpretação de dados. No terceiro setor estão os 
prestadores de serviços. No bloco das atividades chaves do marketing os 7p's são distribuídos conforme o setor de atividade e descrito na revisão de literatura.

Figura 3. O modelo de referência da IoT para o marketing (7 P's x Modelo de referência IoT)

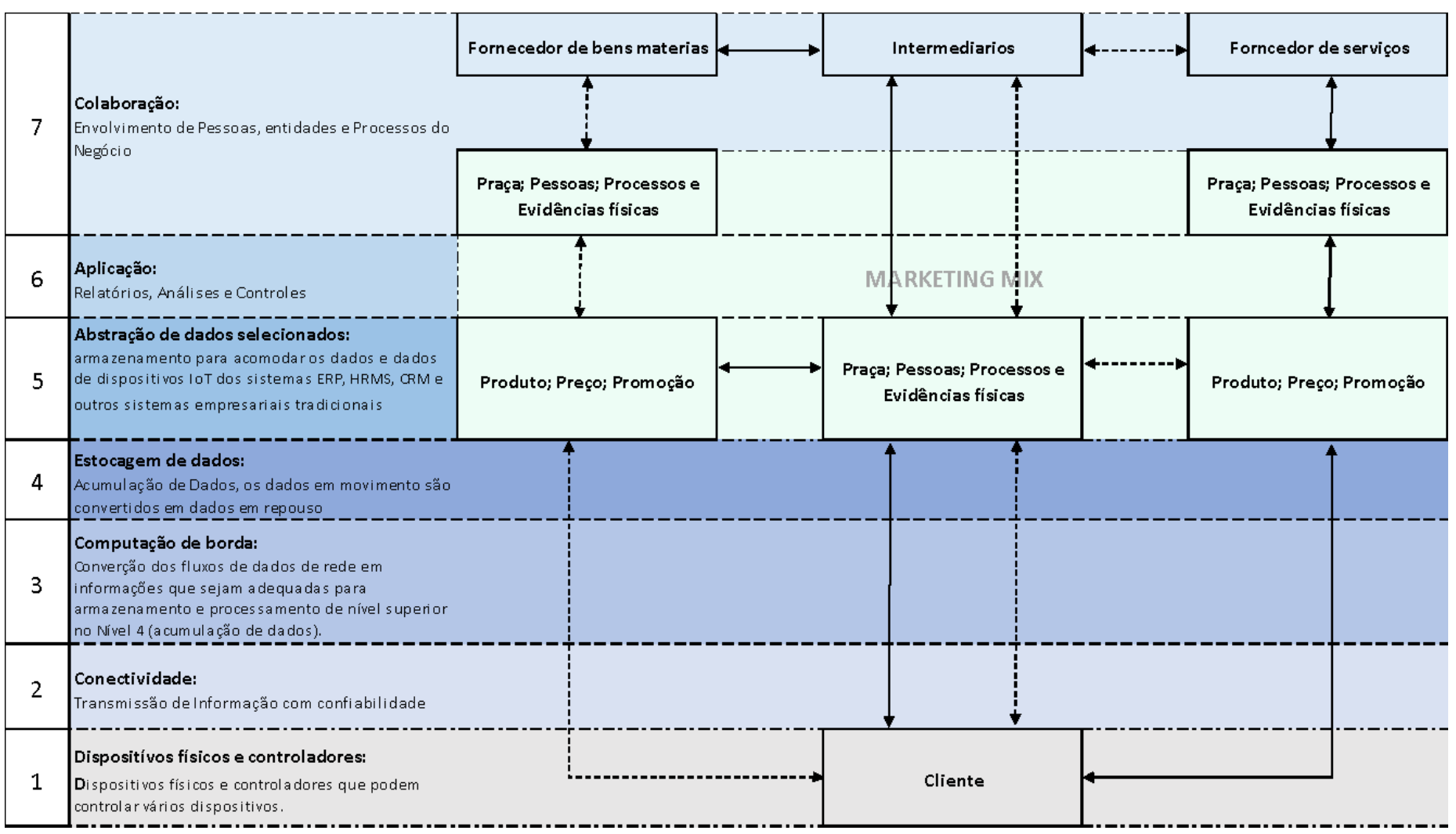

Rota frequente

Rota menos frequente

Foi utilizada uma metodologia de pesquisa teórica para identificar o modelo de mix marketing em modelos de negócios IoT. Subsequentemente, destacados os níveis de aplicação no ambiente de marketing e tipos específicos que são considerados significativamente mais importantes do que outros para o desenvolvimento de estratégia de marketing no ambiente IoT.

Desta maneira, pode-se localizar o "mix marketing" dentro do contexto das sete camadas da IoT. O conjunto do marketing mix representado em verde, situa-se na fronteira do nível 4 com o nível 5, onde acontece a acumulação e a abstração de dados relevantes para a estratégia de marketing. O cliente ou usuário, situa-se no nível 1 (recolhimento de dados), ou seja no mesmo nível que os dispositivo e controladores. A massa de informação obtida é tratada e selecionada para cada tipo de estratégia e setor. Os níveis 2, 3 e 4 são claramente explorados pelos intermediários e prestadores de serviços. Nestes níveis estão todas as atividades de conectividade, conversão e estocagem de dados. Já os produtores atuam majoritariamente nos níveis 6 e 7 (Aplicação e Colaboração), com a exceção dos negócios completamente verticalizados.

É importante notar que as empresas foram incluídas dentro do fluxo de informação dos 7P's pois no contexto da IoT as informações tendem a fluir nos dois sentidos, já que são obtidas do cliente, retornando na forma de posicionamento estratégico de marketing, como descreve o 
modelo tradicional dos 7P's. As informações extraídas de dentro da empresa, também são tratadas e classificadas para o uso em treinamentos e marketing institucional.

Este trabalho pode ser mais um passo para a abertura des discussões e debates adicionais. Neste artigo em especial foram discutidos aspectos teóricos, metodológicos e gerenciais dos processos de marketing e IoT, dando-lhe assim uma visão de geral da IoT no marketing. No entanto, se fazem necessários outros trabalhos para entender as implicações da IoT. Com o objetivo de ampliar o escopo do trabalho aqui apresentado e de encorajar estudos futuros a examinar essas questões de "marketing IoT" de forma mais ampla, com trabalhos empíricos, comportamentais e analíticos, aqui estão alguns fatores fundamentais para o marketing no contexto da IoT , tais como colocam em evidência Nguyen e Simkin (2017):

- Como as empresas devem desenvolver canais de IoT e estratégias de comunicação totalmente integrados para refletir melhor a ampla variedade de opções de IoT?

- Como as empresas IoT devem avaliar a consistência das mensagens em vários pontos de contato?

- Como a eficácia do marketing pode ser medida em um sistema integrado de comunicação IoT?

- Quanto controle as empresas têm sobre seus clientes neste ambiente?

- Quanto controle devem exercer sobre seus clientes com tantos dados disponíveis?

- Qual é a relação entre o CRM (Customer Relationship Management) e o IoT em um ambiente tão voltado para os dados?

- Como as empresas devem desenvolver relacionamentos, alianças e parcerias em um ambiente de IoT?

- Que fatores afetam o envolvimento do consumidor com as empresas? Como as empresas podem usar o IoT para projetar e desenvolver uma melhor experiência do cliente?

- Quais são as métricas e estratégias de marketing ideais que as empresas devem empregar com o IoT?

- Como os anúncios, promoções e outras comunicações personalizadas são processados pelos consumidores através do IoT em comparação com os seus homólogos tradicionais?

- Quais são os custos e benefícios das empresas que adotam o IoT para otimizar o marketing?

Todas estas questões serão objeto de uma pesquisa que será realisada na empresa Petro-Canadá, em Montreal, no Canadá, onde será aplicado o modelo sugerido no segmento das estações de serviços, empresa em que o relacionamento com o cliente, o intercâmbio de infomações e a atravatividade comercial são de fundamental importância, para o crescimento. O uso do marketing mix no contexto da IoT tem um enorme potencial nesta empresa que tem sua estrutura completamente verticalizada desde a extração do petróleo até o ponto de venda.

\section{CONCLUSÃO}

O futuro da IoT é imprevisível, mas isso já está beneficiando uma nova geração de empresas na identificação de suas estratégias de marketing. O acesso à internet a partir de dispositivos móveis e a capacidade de integrar informações, experiências e opiniões, nos permite avanços incríveis no conhecimento do mercado alvo e ações de marketing. 
Este artigo apresentou o potencial da Internet de coisas "IoT" para o mercado atual e o marketing orientado para o valor e para uma relação mais interativa com os clientes através do contexto da IoT.

A integração de todos os níveis e a participação do cliente no marketing agora é possível como resultado dos meios de identificação integrados nos dispositivos eletrônicos e nos telefones celulares. Da mesma forma que a integração na Internet dos telefones inteligentes foi melhorada nos últimos anos, será integrada aos produtos e serviços com o objetivo de facilitar e simplificar a interação dos clientes com as empresas.

\section{REFERÊNCIAS}

A. GLUHAK, S. KRCO, M. NATI, D. PFISTERER, N. Mitton, and T. Razafindralambo, "A survey on facilities for experimental internet of things research," IEEE Communications Magazine, vol. 49, no. 11, pp. 58-67, (2011).

A. GHOSH, S.K. DAS, Coverage and connectivity issues in wireless sensor networks: a survey, Pervasive and Mobile Computing 4 303-334. (2008).

A. JUELS, "RFID security and privacy: a research survey, IEEE Journal on Selected Areas in Communications". (2006).

AMA - American Marketing Association "Mahajan Award for Lifetime Contributions to Marketing Strategy Research," http://ama-academics.communityzero.com/elmar?go = t990773. (2009).

ASHTON, K. “That 'internet of things' thing”. RFiD Journal, 22(7), 97-114. (2009).

ATZORI, L., IERA, A., \& MORABITO, G. "The internet of things: A survey". Computer Networks, 54 (15), 2787-2805. doi:10.1016/j.comnet.2010.05.010 (2010).

BANG NGUYEN \& LYNDON SIMKIN. "The Internet of Things (IoT) and marketing: the state of play, future trends and the implications for marketing". Journal of Marketing Management, 33:1-2, 1-6, DOI: 10.1080/0267257X.2016.1257542. (2017).

BALAJI, M. S. AND S. K. ROY. "Value co-creation with Internet of things technology in the retail industry." Journal of Marketing Management 33(1-2): 7-31. (2017).

BI, Z., XU, L. D., \& WANG, C. "Internet of Things for enterprise systems of modern manufacturing". IEEE Transactions on Industrial Informatics, 10(2), 1537-1546. doi:10.1109/ TII.2014.2300338 (2014).

BITNER, M.J., "Evaluating service encounters: the effect of physical surroundings and employee responses". Journal of Marketing, Vol. 54, p. 69-82. (1990)

BOHLI, J. M., SORGE, C., \& WESTHOFF, D. "Initial observations on economics, pricing, and penetration of the internet of things market". ACM SIGCOMM Computer Communication Review, 39(2), 50-55. (2009).

BOOMS, B.H. AND BITNER, M.J., "Marketing strategies and organization structures for service firms". Donnelly, J.H. and George, W.R. (Eds), Marketing of Services, American Marketing Association, Chicago, IL, pp. 47-51. (1981).

BUTTLE, F. "Marketing services", in Jones, P. (Ed.), Management in Service Industries, Pitman, London, pp. 235-59. (1989).

CISCO "Building the internet of things" World Forum on Internet-of-Things Conference, (2014).

CISCO "The Internet of Things Reference Model" World Forum on Internet-of-Things Conference, (2014). 
CHASE, R. AND GARVIN, D.A. "The service factory", Harvard Business Review, July/August, pp. 61-9. (1989).

CHENG, L.C.; MELO FILHO, L. R. "Platform Conceptual Model In QFD For Generic Drug". Product: Management \& Development, v8, n.1, p.3-16, (2010).

CHUAH, S. H.-W., RAUSCHNABEL, P. A., KREY, N., NGUYEN, B., RAMAYAH, T., \& LADE, S. "Wearable technologies: The role of usefulness and visibility in smartwatch adoption. Computers in Human Behavior", 65, 276-284. doi:10.1016/j.chb.2016.07.047 (2016).

CONOLE, G., M. DE LAAT, T. DILLON AND J. DARBY. "'Disruptive technologies', 'pedagogical innovation': What's new? Findings from an in-depth study of students' use and perception of technology." computers \& Education 50(2): 511-524. (2008)

COWELL, D. “The Marketing of Services, Heinemann”, London. (1984).

CULLITON, J.W. "The Management of Marketing Costs", Division of Research, Graduate School of Business Administration, Harvard University, Boston, MA. (1948).

CHUAH, S. H.-W., RAUSCHNABEL, P. A., KREY, N., NGUYEN, B., RAMAYAH, T., \& LADE, S. "Wearable technologies: The role of usefulness and visibility in smartwatch adoption". Computers in Human Behavior, 65, 276-284. doi:10.1016/j.chb.2016.07.047. (2016).

D.GIUSTO, A. IERA, G.MORABITO, AND L. ATZORI (eds.). "The Internet of Things: 20th Tyrrhenian Workshop on Digital Communications, Springer, Berlin, Germany, (2010).

DHOLAKIA, N., \& REYES, I. Virtuality as place and process. Journal of Marketing Management, 29(13-14), 1580-1591. http://dx.doi.org/10.1080/0267257X.2013.834714 (2013).

E. WELBOURNE, L. BATTLE, G. COLE. "Building the internet of things using RFID: the RFID ecosystem experience". IEEE Internet Computing, vol. 13, no. 3, pp. 48-55, (2009).

FREY, A.W. Advertising, 3rd ed., The Ronald Press, New York, NY. (1961).

GARTNER. Gartner says 6.4 billion connected "things" will be in use in 2016, up 30 percent from 2015. Press Release, STAMFORD, Conn., November 10, 2015. Retrieved in January 20, 2017, from http://www.gartner.com/newsroom/id/3165317. (2015).

GUMMESSON, E. "The new marketing - developing long term interactive relationships", Long Range Planning, Vol. 20 No. 4, pp. 10-24. (1987).

H. SUNDMAEKER, P. GUILLEMIN, P. FRIESS, S. WOELFFLÉ. "Vision and challenges for realising the Internet of Things". Cluster of European Research Projects on the Internet of Things-CERP IoT, (2010).

HALLER S, KARNOUSKOS S, SCHROTH C. "The Internet of Things in an enterprise context". Future Internet Symp 5468:14-28. (2009).

HOFFMAN, D. L., \& NOVAK, T. P. "Emergent experience and the connected consumer in the smart home assemblage and the internet of things". Retrieved from http://ssrn.com/abstract= 2648786 doi: $10.2139 /$ ssrn.2648786 (2015).

HSU, C.-L., \& LIN, -C.-C. "An empirical examination of consumer adoption of Internet of Things services: Network externalities and concern for information privacy perspectives". Computers in Human Behavior, 62, 516-527. doi:10.1016/j.chb.2016.04.023. (2016).

I.F. AKYILDIZ, W. SU, Y. Sankarasubramaniam, E. Cayirci. "Wireless sensor networks: a survey". Computer Networks 38, 393-422. (2002).

INKELES, A. "What is sociology? An introduction to the discipline and profession". Englewood Cliffs: Prentice-Hall. (1964).

INTERNATIONAL SYMPOSIUM ELECTRONICS IN MARINE. Grgić, M., Božek, J., Grgić, S. Institute of Electrical and Electronics Engineers. Institute of Electrical and Electronics Engineers., \& 
European Association for Speech, Signal and Image Processing. Proceedings ELMAR-2009: 51st International Symposium ELMAR-2009, 28-30 September 2009, Zadar, Croatia. Zadar: Croatian Society Electronics in Marine. (2009).

J. BELISSENT. "Getting clever about smart cities: new opportunities require new business models" FORRESTER RESEARCH, (2010).

JONES, R., SUORANTA, M., \& ROWLEY, J. "Strategic network marketing in technology SMEs". Journal of Marketing Management, 29(5-6), 671-697. doi:10.1080/0267257X.2013.797920 (2013).

KORTUEM, G., KAWSAR, F., SUNDRAMOORTHY, V., \& FITTON, D. "Smart objects as building blocks for the Internet of Things". IEEE Internet Computing, 14(1), 44-51. doi:10.1109/MIC.2009.143 (2010).

KOTLER, P. "Marketing Management”. 3rd ed., Prentice-Hall, Englewood Cliffs, NJ, p. 60. (1976)

KOTLER, P. "Megamarketing”. Harvard Business Review, Vol. 64, March/April, pp. 117-24. (1986).

KOTLER, P. AND ARMSTRONG, G. "Principles of Marketing", 4th ed., Prentice-Hall, Englewood Cliffs, N.J. (1989).

LAZER, W., CULLEY, J.D. AND STAUDT, T. "The concept of the marketing mix", in Britt, S.H. (Ed.), Marketing Manager's Handbook, The Dartnell Corporation, Chicago, IL, pp. 39-43. (1973).

LAZER, W. AND KELLY, E.K. "Managerial Marketing: Perspectives and Viewpoints". Richard D. Irwin, Homewood, IL. (1962).

LEVITT, T. "The industrialization of service", Harvard Business Review, Vol. 54, September/October, pp. 63-74. (1976).

M. TORY, T. MOLLER. "Rethinking visualization: a high-level taxonomy", in: IEEE Symposium on Information Visualization, 2004, INFOVIS 2004, pp. 151-158. (2004).

M. ZORZI, A. GLUHAK, S. LANGE, A. BASSI. "From today's Intranet of Things to a future Internet of Things: a wireless- and mobility-related view". IEEE Wireless Communications 17, 43-51. (2010)

MA, T., \& ZHANG, C. "On the Disruptive Potentials in Internet of Things". In Parallel and Distributed Systems (ICPADS), 2011 IEEE 17th International Conference on (pp. 857-859). IEEE. (2011).

MCCARTHY, E.J. “Basic Marketing”, Richard D. Irwin, Homewood, IL. (1964).

MCCARTHY, E.J. AND PERREAULT, W.D. JR. "Basic Marketing", 9th ed., Richard D. Irwin, Homewood, IL. (1987).

N. HONLE, U.P. KAPPELER, D. NICKLAS, T. SCHWARZ, M. GROSSMANN. "Benefits of integrating meta data into a context model". pp. 25-29. (2005).

NGUYEN, B., \& DE CREMER, D. "The fairness challenge of the Internet of Things." European Business Review, (pp. 31-33) http://www.europeanbusinessreview.com/?p= 8588. (2016).

O'CONNOR, G. C., \& VERYZER, R. W. "The nature of market visioning for technology-based radical innovation”. Journal of Product Innovation Management, 18(4), 231-246. (2001).

PORTER, M. E. "From competitive advantage to competitive strategy". Harvard Business Review, 65, 43-59. (1987).

PORTER, M. E. "What is strategy?". Harvard Business Review, 74, 61-78. (1996).

PORTER, M. E., \& HEPPELMANN, J. E. "How smart, connected products are transforming competition". Harvard Business Review, November Issue, Retrieved February 8, 2015, from https://hbr.org/2014/11/how-smart-connected-products-are-transforming-competition. (2014).

PRIDE, W.M. AND FERRELL O.C. "Marketing: Concepts and Strategies". 6th ed., Houghton-Mifflin, Boston, MA. (1989). 
R. BUYYA, C.S. YEO, S. VENUGOPAL, J. BROBERG, I. BRANDIC. "Cloud computing and emerging IT platforms: vision, hype, and reality for delivering computing as the 5th utility". Future Generation Computer Systems 25, 599-616. (2009).

RAFIQ, M. AND P. K. AHMED. "Using the 7Ps as a generic marketing mix: an exploratory survey of UK and European marketing academics". Marketing Intelligence \& Planning 13(9): 4-15. (1995).

RUI H, YIP GS. "Foreign acquisitions by Chinese firms: a strategic intent perspective". J World Bus 43(2):213-226 (2008).

S. TILAK, N. ABU-GHAZALEH, W. HEINZELMAN. "A taxonomy of wireless microsensor network models". ACM Mobile Computing and Communications Review 6, 28-36. (2002).

SHAPIRO, B.P. "Rejuvenating the marketing mix". Harvard Business Review, September/October, pp. 28-34. (1985).

SICARI, S., RIZZARDI, A., GRIECO, L. A., \& COEN-PORISINI, A. "Security, privacy and trust in Internet of Things: The road ahead". Computer Networks, 76, 146-164. doi:10.1016/j.comnet.2014.11.008. (2015).

SLETTEMEÅS, D. "RFID-the "Next Step" in consumer-product relations or Orwellian nightmare? challenges for research and policy". Journal of Consumer Policy, 32, 219-244. doi:10.1007/s10603009-9103-z. (2009).

WARD PT, DURAY R. "Manufacturing strategy in context: environment, competitive strategy and manufacturing strategy". J Oper Manag 18(2):123-138. (2000).

WEBSTER, F.E. JR. "Management science in industrial marketing". Journal of Marketing, Vol. 42, January, pp. 21-7. (1978).

WEBSTER, F.E. JR. "Industrial Marketing Strategy”. 2nd ed., John Wiley \& Sons, New York, NY. (1984).

WEBSTER, F.E. JR AND WIND, Y. "A general model for understanding organizational buying behaviour". Journal of Marketing, Vol. 36, April, pp. 12-19. (1972).

WELBOURNE. "Building the Internet of Things using RFID: the RFID ecosystem experience". Internet Computing IEEE 13(3):48-55 (2009).

Y. BENDAVID AND H. BOECK, "Using RFID to improve hospital supply chain management for high value and consignment items". Procedia Computer Science, vol. 5, pp. 849-856. (2011).

Y. SANG, H. SHEN, Y. INOGUCHI, Y. TAN, N. XIONG, "Secure Data Aggregation in Wireless Sensor Networks: A Survey", pp. 315-320. (2006). 